\title{
Análise da inserção de agricultores familiares prioritários nas chamadas públicas da alimentação escolar
}

\author{
Analysis of the insertion of priority family farmers in public calls for school food \\ Análisis de la inserción de agricultores familiares prioritarios en las convocatorias públicas de \\ alimentación escolar
}

Recebido: 18/03/2021 | Revisado: 26/03/2021 | Aceito: 30/03/2021 | Publicado: 10/04/2021

Cassia Bonfim de Oliveira

ORCID: https://orcid.org/0000-0003-0819-7728 Universidade Federal de Sergipe, Brasil

E-mail: cassia.nutri6@gmail.com

Silvia Eloíza Priore

ORCID: https://orcid.org/0000-0003-0656-1485 Universidade Federal de Viçosa, Brasil E-mail: sepriore@ufv.br

Renata Lopes de Siqueira

ORCID: https://orcid.org/0000-0002-4776-146X Universidade Federal de Sergipe, Brasil E-mail: renata.educacao@gmail.com

\begin{abstract}
Resumo
Introdução: A Lei no 11.947/2009 exigiu a aplicação de, no mínimo, 30\% dos recursos repassados pelo governo federal, na compra de alimentos da Agricultura Familiar (AF) para a alimentação escolar, provenientes, preferencialmente, do âmbito local, priorizando as comunidades vulneráveis, com gêneros orgânicos e agroecológicos e os grupos de fornecedores com participação formal. Objetivo: o presente estudo realizou uma série histórica das Chamadas Públicas (CP), observando a ocorrência da aquisição de gêneros alimentícios conforme critérios de priorização estabelecidos na legislação do PNAE no município de São Cristóvão/SE. Metodologia: Trata-se de um estudo de caso, com análise documental. Analisou-se as $\mathrm{CP}$ e contratos de vendas referentes às aquisições dos produtos da AF no período de 2010 a 2020. Resultados: Contrariando a Lei, evidenciou-se que ao longo da década de estudo, os agricultores familiares residentes no município tiveram menor participação nas CP que os demais fora do território local. Observou-se menor participação das comunidades vulneráveis no programa e a falta de acesso de produtores de gêneros orgânicos e agroecológicos. Em concordância com a legislação, registrou-se maior participação dos grupos formais de agricultores em todos os processos de CP. Conclusão: Ações intersetoriais são necessárias visando facilitar o acesso e garantir a participação desses grupos nas $\mathrm{CP}$, para que o programa enquanto política pública, garanta a segurança alimentar e nutricional de escolares e comunidades locais.
\end{abstract}

Palavras-chave: Alimentação escolar; Agricultura sustentável; Direito humano à alimentação adequada; Segurança alimentar e nutricional.

\begin{abstract}
Introduction: Law $\mathrm{n}^{\circ} 11.947 / 2009$ required the application of at least $30 \%$ of the funds transferred by the federal government, in the purchase of food from Family Agriculture (AF) for school meals, originating, preferably, from the local level, prioritizing the vulnerable communities, with organic and agroecological genera and groups of suppliers with formal participation. Objective: the present study carried out a historical series of Public Calls (PC), observing the occurrence of the acquisition of foodstuffs according to the prioritization criteria established in the PNAE legislation in the municipality of São Cristóvão/SE. Methodology: This is a case study, with documentary analysis. PC and sales contracts related to the acquisition of AF products in the period from 2010 to 2020 were analyzed. Results: Contrary to the Law, it was evident that throughout the decade of study, family farmers living in the municipality had less participation in the PC than the others outside the local territory. There was less participation of vulnerable communities in the program and the lack of access for producers of organic and agroecological products. In accordance with the legislation, there was a greater participation of formal groups of farmers in all PC processes. Conclusion: Intersectoral actions are necessary to facilitate access and ensure the participation of these groups in PCs, so that the program, as a public policy, guarantees food and nutrition security for schoolchildren and local communities.
\end{abstract}

Keywords: School feeding; Sustainable agriculture; Human right to adequate Food; Food and nutrition security. 


\begin{abstract}
Resumen
Introducción: La Ley $\mathrm{n}^{\circ}$ 11.947/2009 requirió la aplicación de al menos el 30\% de los fondos transferidos por el gobierno federal, en la compra de alimentos a la Agricultura Familiar (AF) para las comidas escolares, con origen, preferentemente, del nivel local, priorizando las comunidades vulnerables, con géneros orgánicos y agroecológicos y grupos de proveedores con participación formal. Objetivo: el presente estudio realizó una serie histórica de Convocatorias Públicas (CP), observando la ocurrencia de la adquisición de alimentos según los criterios de priorización establecidos en la legislación del PNAE en el municipio de São Cristóvão/SE. Metodología: Se trata de un estudio de caso, con análisis documental. Se analizaron CP y contratos de venta relacionados con la adquisición de productos AF en el período de 2010 a 2020. Resultados: Contrario a la Ley, se evidenció que a lo largo de la década de estudio, los agricultores familiares residentes en el municipio tuvieron menor participación en el CP que los demás fuera del territorio local. Hubo menor participación de comunidades vulnerables en el programa y falta de acceso para productores de productos orgánicos y agroecológicos. De acuerdo con la legislación, hubo una mayor participación de los grupos formales de agricultores en todos los procesos de CP. Conclusión: Son necesarias acciones intersectoriales que faciliten el acceso y aseguren la participación de estos grupos en los CP, para que el programa, como política pública, garantice la seguridad alimentaria y nutricional de los escolares y las comunidades locales.
\end{abstract}

Palabras clave: Alimentación escolar; Agricultura sostenible; Derecho humano a una alimentación adecuada; Seguridad alimentaria y nutricional.

\title{
1. Introdução
}

O Programa Nacional de Alimentação Escolar (PNAE), objetiva contribuir para o crescimento e o desenvolvimento biopsicossocial, a aprendizagem, o rendimento escolar e a formação de hábitos alimentares saudáveis dos escolares, conforme proposto na Lei ${ }^{\circ}$ 11.947, de 2009. Ele constitui-se em uma estratégia de Segurança Alimentar e Nutricional (SAN) consoante aos princípios do Direito Humano à Alimentação Adequada (DHAA) (Silva Sa et. al, 2017).

Como estratégia de garantia da SAN e do DHAA, a referida Legislação definiu que, do total dos recursos financeiros repassados pelo Fundo Nacional do Desenvolvimento da Educação (FNDE), no âmbito do PNAE, no mínimo 30\% deverão ser utilizados na aquisição de gêneros alimentícios diretamente da Agricultura Familiar (AF) e do empreendedor familiar rural ou de suas organizações, priorizando os assentamentos da reforma agrária, as comunidades tradicionais indígenas e comunidades quilombolas (Lei n 11.947, 2009; Nascimento; Nascimento \& Oliveira, 2019), pois o programa reconhece as condições de vulnerabilidade e precariedade socioeconômica dessas minorias sociais, que, atuando como produtores agrícolas, enfrentam grande dificuldade de inserção e comercialização de sua produção no mercado institucional e não institucional (Resolução ${ }^{\circ}$ 10, 2018; Nogueira et al., 2016).

Também foi determinado que a aquisição dos produtos da AF deveria ocorrer via Chamada Pública (CP), uma alternativa ao modelo convencional de compra via processo licitatório, que dificultava a comercialização dos produtos da AF em função das condições desiguais de competitividade das demais categorias de fornecedores (Resolução $n^{\circ}$ 10, 2018).

A Resolução/CD/FNDE n 38 publicada em 2009 não definiu os critérios de seleção, priorizou apenas as propostas do município, e definiu, que sempre que possível, os alimentos orgânicos e agroecológicos fossem priorizados (Resolução/CD/FNDE $n^{\circ} 38,2009$ ). No entanto, devido às dificuldades relatadas pelos produtores quanto ao custo para a produção e comercialização destes produtos, na Resolução/CD/FNDE n 26 de 2013 ficou instituída que, se as Entidades Executoras (EEx) priorizassem a aquisição desses gêneros, poderia acrescer os preços em até $30 \%$ em relação aos preços estabelecidos para produtos convencionais (Resolução/CD/FNDE nº 26, 2013).

Posteriormente, a Resolução/CD/FNDE $n^{\circ} 4$ publicada em 2015, alterou a redação dos $\operatorname{artigos} 25$ a 32 da Resolução/CD/FNDE nº 26/2013, adotando para a seleção dos projetos de venda a seguinte sequência: grupo de projeto locais, de territórios rurais, de projetos do estado e grupos de propostas do país, respectivamente. Os critérios de priorização permaneceram os mesmos (I- comunidades vulneráveis; II- gêneros orgânicos e agroecológicos e III- participação formal dos agricultores) (Resolução/CD/FNDE nº 4, 2015).

Em 2020, a Resolução $\mathrm{n}^{\circ} 6$ do FNDE, revogou as resoluções anteriores, e os grupos de território rural foram 
substituídos por grupos de Regiões Geográficas Imediatas e Grupos de Regiões Geográficas intermediárias. Outra alteração realizada refere-se às Cooperativas Centrais da $\mathrm{AF}$, as quais faziam parte dos grupos formais com as associações, passando a ser um grupo isolado e ocupar o quarto lugar no critério "modalidade de participação" (Resolução nº 6, 2020).

Entretanto, estudos realizados por Noronha (2013) em Capão do Leão - RS e Messias (2018) em três municípios do estado de São Paulo, apontaram baixo acesso de agricultores familiares que fazem parte dos grupos prioritários estabelecidos em Lei nas aquisições do programa. Assim, suspeita-se que no processo de compra de gêneros alimentícios da AF pelos entes públicos, prevalecem a aquisição de produtos dos grupos não prioritários, privilegiando grandes cooperativas constituídas por produtores de base convencional, não residentes na área de abrangência local ou territorial.

Deste modo, o objetivo do presente artigo é realizar uma série histórica das chamadas públicas de aquisição de gêneros alimentícios da agricultura familiar para a alimentação escolar no município de São Cristóvão-SE, segundo critérios de priorização estabelecidos na legislação do PNAE.

Não obstante, os resultados da presente análise permitirão revelar se o que foi estabelecido na legislação tem sido executado na prática, assim como, poderão suscitar questões de reflexão para nortear novos estudos e realizar uma análise crítica acerca dos procedimentos de monitoramento e exigibilidade do cumprimento das medidas resolutivas instituídas com a finalidade de assegurar o desenvolvimento local, a sustentabilidade ambiental e, sobretudo, a garantia do DHAA e SAN dos agricultores familiares e escolares da rede pública de ensino. Ademais, não foram encontrados estudos realizados sobre essa abordagem.

\section{Metodologia}

Trata-se de um estudo de abordagem qualitativa descritiva, tendo como método de pesquisa o estudo de caso sobre o processo de aquisição dos gêneros alimentícios provenientes da AF pelo município de São Cristóvão, antiga capital sergipana, no período de 2010 a 2020.

Segundo Prodanov e Freitas (2013), o estudo de caso é "uma categoria de investigação que tem como objeto o estudo de uma unidade de forma aprofundada podendo tratar-se de um sujeito, de um grupo de pessoas, de uma comunidade, dentre outras (Prodanov \& Freitas, 2013, pg.60). Nessa modalidade de pesquisa, busca-se a investigação de um caso específico, bem delimitado, contextualizado em tempo e lugar, o que permite realizar uma busca circunstanciada de informações (Pereira et al., 2018; Ventura, 2007).

O procedimento utilizado foi a pesquisa documental, que visa compreender a realidade social de forma indireta mediante análise de inúmeros tipos de documentos (Silva et al., 2009).

Foram utilizados os editais de $\mathrm{CP}$ referentes à aquisição de gêneros alimentícios provenientes da $\mathrm{AF}$ e os contratos de venda delas decorrentes. Parcela dos documentos foram obtidos no site institucional do município (https://www.saocristovao.se.gov.br/). Aqueles que não estavam disponíveis virtualmente, foram adquiridos por meio de contato direto com a comissão de licitação municipal. Informações complementares foram obtidas pela nutricionista responsável técnica do programa e pela presidente do Sindicato de Trabalhadores Rurais do município por contato telefônico.

Para a coleta de dados nos editais de CP e contratos de vendas, foi elaborado um checklist tendo as variáveis qualitativas definidas com base nos dispositivos legais.

Inicialmente, visando caracterizar o município, foi realizado um levantamento de dados secundários do banco de dados estatísticos do Instituto Brasileiro de Geografia e Estatística (IBGE). Foram coletados os dados sobre o censo demográfico municipal, estimativa da população residente em 2020, mapas da pobreza e desigualdade, trabalho e rendimento. Ademais, foram coletados dados da produção agrícola e do censo agropecuário do município.

Na sequência, nos editais publicados a partir de 2015, foi verificada a presença dos itens propostos no modelo 
disponibilizado na Resolução CD/FNDE n ${ }^{\circ}$ 4/2015. A adoção desta resolução como referência, justifica-se por ser a normativa legal vigente do período entre 2015 e 2020.

Em todos os editais de CP do período 2010-2020, foram analisados os dados cadastrais da EEx responsável, legislação base adotada, período de vigência das $\mathrm{CP}$, a presença e especificação dos critérios de procedência, seguida da observância da descrição dos critérios de priorização segundo situação de vulnerabilidade social (comunidades tradicionais indígenas, quilombolas ou Assentados da Reforma Agrária); modo de produção (orgânico, agroecológico ou convencional) e; a modalidade de participação (grupo formal, informal ou individual).

Posteriormente, os contratos de venda foram analisados para identificar se os agricultores familiares contratados estavam em consonância ou não com os critérios de priorização originalmente previstos nas respectivas CP. Complementarmente, foi averiguada a presença da lista de gêneros alimentícios ofertados no intuito de identificar se o modo de produção de cada alimento estava explícito, quando esta informação não estava descrita nas cláusulas de contratação. Igualmente, foi observado se a legislação de base adotada nos contratos de venda estava compatível com a legislação vigente, conforme período de execução previsto na CP.

Todos os dados coletados foram sistematizados em planilhas no programa Microsoft Excel e realizada a análise estatística descritiva.

Esta pesquisa faz parte de um projeto maior, o qual foi aprovado pelo comitê de ética em pesquisa com seres humanos sob parecer $n^{\circ} 3.844 .532$.

\section{Resultados e Discussão}

\section{Caracterização do local de estudo}

Segundo os dados do último censo demográfico publicados pelo IBGE em 2010, o município de São Cristóvão, Sergipe, apresentou uma população de 78.864 habitantes. Sua população estimada para 2020 foi de 91.093 habitantes, demonstrando necessidade de políticas públicas. O município apresentou uma área de $438,037 \mathrm{~km}^{2}$, densidade demográfica de 180,52 habitantes $/ \mathrm{km}^{2}$ e 84,5 \% da população residente em área urbana (Instituto Brasileiro de Geografia e Estatística, 2010; IBGE, 2020).

Observou-se que parcela da população do município vive em condições de insegurança alimentar e nutricional, conforme dados obtidos no site do IBGE, corroborando com o estudo de Santos (2018).

O Produto Interno Bruto (PIB) per capita do município ( $\$$ \$ 10.061.71), em 2018, foi maior que em 2015 (R\$ 9.912,72). Entretanto, muito abaixo da média da capital do estado (R \$26.622,38) (IBGE, 2018).

O município que sedia a Universidade Federal de Sergipe (UFS), apesar de ter sido a primeira capital do estado, possui uma situação socioeconômica que demanda atenção visto que os dados do último censo indicam que a incidência de pessoas pobres é de 57,1\% (IBGE, 2003), Índice de Desenvolvimento Humano Municipal (IDHM) de 0,662 (IBGE, 2010), a proporção de pessoas pobres, ou seja, com renda domiciliar per capita inferior a $R \$ 140,00$ no município é de 22,9\% e o Índice de Gini de 0,42 (IBGE, 2003). Destaca-se que este último índice é um instrumento usado para medir o grau de concentração de renda. Ele aponta a diferença entre os rendimentos dos mais pobres e dos mais ricos. Numericamente, varia de 0 a 1 , sendo que o limite inferior a 0 representa a situação de total igualdade e, limite superior 1 significa completa desigualdade de renda, ou seja, uma só pessoa detém toda a renda do lugar.

Em 2018, o salário médio mensal era de 3,6 salários mínimos. No entanto, observa-se um situação de desigualdade, visto que a proporção de pessoas ocupadas em relação à população total era apenas de 16,1\%. Ainda, considerando domicílios com rendimentos mensais de até meio salário mínimo por pessoa, 41,6\% da população apresentavam-se nessas condições, o que demonstra situação de pobreza. Neste mesmo ano, 61,7\% dos domicílios não possuía esgotamento sanitário adequado 
(IBGE, 2018).

Em relação ao setor agropecuário, o Censo Agropecuário de 2017 demonstrou que o município possuía 946 estabelecimentos agropecuários: deste total, 63,5\% (601) eram estabelecimentos da $\mathrm{AF}$ e 36,4\% (345) eram de estabelecimentos da agricultura não familiar, apresentando aumento de 13,6\% destes comparados ao ano anterior (IBGE, 2017).

A produção agrícola com destaque na agricultura está o da cana-de-açúcar, coco-da-baía, mandioca, banana, milho e feijão (IBGE, 2019). Destes, a mandioca, o milho e o feijão são os gêneros tradicionais produzidos pelo município.

\section{Levantamento das Chamadas Públicas e contratos}

A integralidade das CP e dos contratos formalizados referentes a aquisição de gêneros alimentícios da AF para o PNAE no município de São Cristóvão-SE, aconteceram regularmente no início de cada ano e tiveram vigência até o último dia do ano em exercício. Entretanto, embora seja positiva a ocorrência dessa regularidade, devido aos aspectos burocráticos e a organização das etapas do processo de $\mathrm{CP}$, alguns estudos destacam que a realização anual das CP pode ocasionar efeitos negativos.

De acordo com Assis, França e Coelho (2019) e Triches, Barbosa e Silvestri (2016), para que os preços sejam atendidos conforme a sazonalidade da produção dos alimentos, faz-se necessário que as CP sejam realizadas mais de uma vez por ano, pois durante o ano há variações entre chuva e seca, safra e entressafra, provocando alterações nos preços dos produtos do mercado local. Quando as CP ocorrem anualmente, os preços estão fixos nos editais, e consequentemente, desatualizados em relação ao valor do produto no mercado local devido às variações supracitadas.

Foram analisados 11 editais de CP compreendidos entre 2010 e 2020, tendo sua vigência coincidindo com o ano em exercício. Deste total, quatro não foram publicizados após a homologação (Tabela 1), mas foram obtidos com a nutricionista Responsável Técnica (RT) do PNAE. Segundo a profissional, a não publicização se deve ao fato de que na época, o setor responsável ainda estava em fase de organização operacional, o que dificultou o cumprimento adequado de todas as etapas das CP.

Quanto aos contratos formalizados, foram obtidos um total de 53, conforme mostra a Tabela 1. Não foi possível acessar àqueles referentes aos anos 2010, 2011 e 2015 pelo portal da transparência ou diário oficial do município. Alternativamente, procedeu-se, por via telefônica, solicitações ao órgão municipal responsável pelos contratos, mas não houve retorno. 
Tabela 1. Número obtido de chamadas públicas e contratos realizados pelo município de São Cristóvão/SE, 2010-2020.

\begin{tabular}{cccc}
\hline ANO & CP HOMOLOGADAS & CP PUBLICIZADAS & CONTRATOS PUBLICIZADOS \\
\hline 2010 & 1 & 0 & 0 \\
2011 & 1 & 0 & 0 \\
2012 & 1 & 1 & 3 \\
2013 & 1 & 0 & 3 \\
2014 & 1 & 0 & 4 \\
2015 & 1 & 1 & 1 \\
2016 & 1 & 1 & 5 \\
2017 & 1 & 1 & 8 \\
2018 & 1 & 1 & 10 \\
2019 & 1 & 1 & 9 \\
2020 & 1 & 1 & 10 \\
\hline Total & 11 & 7 & 53 \\
\hline
\end{tabular}

Fonte: Autoras.

De acordo com a Resolução CD/FNDE n 38/2009 e a Resolução CD/FNDE n 26/2013, as CP homologadas e os contratos de venda referentes à aquisição de gêneros alimentícios da AF para o PNAE, devem ser publicados, dentre outros, em local público de ampla circulação e em seu site na internet, quando disponível, para que todos os agricultores familiares e suas organizações tenham conhecimento.

Ainda, a Lei $\mathrm{n}^{\circ}$ 12.527/2011 que regula o acesso às informações, estabeleceu que os órgãos e entidades do poder público deverão assegurar a gestão transparente da informação, propiciando amplo acesso a ela e sua divulgação, assim como, a garantia da sua disponibilidade, autenticidade e integridade, tendo os cidadãos o direito de obter informações de registros ou documentos, produzidos ou acumulados por seus órgãos, ou entidades, recolhidos ou não a arquivos públicos e informações pertinentes à administração do patrimônio público, utilização de recursos públicos, licitação e contratos administrativos (Lei ${ }^{\circ}$ $12.527,2011)$.

Portanto, a não publicização da integralidade das CP homologas e respectivos contratos de venda verificados representa uma situação de descumprimento às Leis $n^{\circ} 12.527 / 2011$ e $\mathrm{n}^{\circ}$ 8.666/93. Esta última instituiu em seu artigo $\mathrm{n}^{\circ} 38$ que, nos processos licitatórios (incluindo a dispensa de licitação), deverão ser juntados, oportunamente, os editais, os comprovantes das publicações do edital resumido, assim como, os atos de adjudicação do objeto e sua homologação (Lei ${ }^{\circ}$ 8.666, 1993).

O Quadro 1 mostra a legislação base tomada como referência em cada edital de CP e contratos de vendas durante o período analisado. Embora a Resolução CD/FNDE n 26 fora publicada em 2013, a resolução anterior poderia ser utilizada como base da CP neste mesmo ano, pois o período de publicação e os trâmites da CP já havia consolidado, justificando a utilização daquela como referência. 
Quadro 1. Legislação utilizada como referência nos editais de Chamada Pública e contratos, São Cristóvão/SE, 2010-2020.

\begin{tabular}{|c|c|c|}
\hline ANO & LEGISLAÇÃO BASE & AVALIAÇÃO \\
\hline 2010 & $\begin{array}{c}\text { Lei no }^{\circ} 11.947 / 2009 \\
\text { Resolução CD/FNDE nº 38/2009 }\end{array}$ & Conforme \\
\hline 2011 & $\begin{array}{c}\text { Lei n }^{\circ} \text { 11.947/2009 } \\
\text { Resolução CD/FNDE nº 38/2009 }\end{array}$ & Conforme \\
\hline 2012 & $\begin{array}{c}\text { Lei no } 11.947 / 2009 \\
\text { Resolução CD/FNDE nº 38/2009 }^{\circ} \text {. }\end{array}$ & Conforme \\
\hline 2013 & 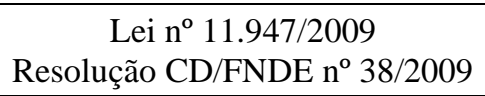 & Conforme \\
\hline 2014 & $\begin{array}{c}\text { Lei no }^{\circ} 11.947 / 2009 \\
\text { Resolução CD/FNDE nº 26/2013 }\end{array}$ & Conforme \\
\hline 2015 & $\begin{array}{c}\text { Lei no }^{\circ} 11.947 / 2009 \\
\text { Resolução CD/FNDE nº }^{26 / 2013}\end{array}$ & Conforme \\
\hline 2016 & $\begin{array}{c}\text { Lei no } 11.947 / 2009 \\
\text { Resolução CD/FNDE nº }^{\circ} 6 / 2013\end{array}$ & Parcialmente conforme \\
\hline 2017 & $\begin{array}{c}\text { Lei n }^{\circ} \text { 11.947/2009 } \\
\text { Resolução CD/FNDE nº 26/2013 }\end{array}$ & Parcialmente conforme \\
\hline 2018 & $\begin{array}{c}\text { Lei no }^{\circ} 11.947 / 2009 \\
\text { Resolução CD/FNDE nº 26/2013 }\end{array}$ & Parcialmente conforme \\
\hline 2019 & $\begin{array}{c}\text { Lei n }^{\circ} \text { 11.947/2009 } \\
\text { Resolução CD/FNDE nº 26/2013 }\end{array}$ & Parcialmente conforme \\
\hline 2020 & $\begin{array}{c}\text { Lei no }^{\circ} 11.947 / 2009 \\
\text { Resolução CD/FNDE n⿳0 26/2013 } \\
\text { Resolução CD/FNDE n }{ }^{\circ} \text { 4/2015 }\end{array}$ & Conforme \\
\hline
\end{tabular}

Fonte: Autoras.

Entre 2016 e 2019, as CP não tomaram como referência a Resolução CD/FNDE nº 4 que fora publicada naquele ano (Quadro 1). Ela deveria estar incluída como referência base, uma vez que a mesma reorganiza os critérios de seleção e priorização dos projetos de venda. Esta resolução foi referenciada apenas no edital do ano 2020.

Observou-se que os editais de CP entre 2015 e 2020 não se adequaram exatamente ao modelo proposto pela Resolução CD/FNDE no 4/2015. Os itens objeto e disposições gerais são os únicos que apareceram idênticos ao modelo proposto. Os itens habilitação do fornecedor e amostras dos produtos apareceram nos editais, porém não foram descritos conforme modelo. Os demais itens sugeridos pela resolução: projeto de venda, critérios de seleção dos beneficiários, local de entrega dos produtos, periodicidade de entrega e documentação, apareceram nos editais como subitens.

O item fonte de recursos não foi descrito em nenhum dos editais analisados. Destaca-se que conhecer a fonte de recursos que serão pagos ao agricultor ou empreendedor familiar, além de demonstrar a transparência pública, reflete maior segurança para estes em vender seus produtos para o mercado institucional, uma vez que há insegurança por parte de alguns produtores em ofertar seus gêneros ao PNAE devido a atrasos nos pagamentos e a crença de que o governo não cumpre com seus compromissos nos prazos estipulados (Marques et al., 2014).

O modelo proposto pela Resolução CD/FNDE $n^{\circ}$ 4/2015, facilita a elaboração da CP e contém as informações elementares, evitando que outras de menor relevância sejam incluídas, em detrimento daquelas. A Resolução $n^{\circ}$ 6/2020, enfatiza ser fundamental o seguimento do modelo, pois o mesmo trata do processo de seleção do fornecedor de gêneros alimentícios de forma objetiva e clara, primando apenas pela inserção de informações obrigatórias por Lei (Resolução ${ }^{\circ}$ 6/2020).

O não seguimento do modelo pode acarretar consequências negativas no processo de aquisição dos gêneros alimentícios da AF, como por exemplo, a possibilidade de descumprimento dos critérios de priorização e do percentual mínimo 
de aquisição da AF, conforme evidenciado cientificamente (Alexandre et al., 2016; Baccarin et al., 2017; Dorneles et al., 2019; Gregolin et al., 2017).

\section{Seleção e priorização dos projetos de venda}

Em relação aos editais de CP realizados entre 2010 e 2013, os critérios de seleção adotados foram apenas a participação em grupo formal e/ou informal. Nestes editais, foi estabelecido que teriam prioridades os grupos de fornecedores formais e de âmbito local. No entanto, os contratos referentes aos anos 2012 e 2013 foram celebrados apenas com grupos formais de outros municípios do estado, como mostra a Tabela 2.

Esta situação contraria as diretrizes e os objetivos do PNAE, no sentido de fortalecer os Circuitos Curtos de comercialização, favorecer a inclusão social e o desenvolvimento local (Aguiar \& Calil, 2018; Alexandre et al., 2016; Gonçalves et al., 2015). Esse achado pode estar associado à falta de conhecimento dos agricultores sobre a CP, visto que não houve publicização nos dois primeiros anos de análise; aos aspectos burocráticos exigidos para acesso dos produtores ao programa, bem como, a falta de preparo técnico dos órgãos e atores responsáveis pela elaboração das CP e ao desconhecimento destes acerca das condicionalidades legais (Castellani et al., 2017; Gregolin, Santos, Felippini, Mateus \& Christoffo, 2017; Rockett et al., 2019; Triches, Simonetti, Cessarino, Baccarin \& Teo, 2019).

Ressalta-se que, previamente às $\mathrm{CP}$, é importante a realização de um mapeamento detalhado dos agricultores familiares da região por parte dos gestores, visando identificar a vocação agrícola do município, a capacidade produtiva e o tipo de produção realizada, assim como, incentivar a maior participação e garantir o acesso desses produtores no processo de CP (Mossman et al., 2017).

Nos anos 2014 e 2015, a EEx utilizou como critérios de seleção nas CP as propostas de grupos formais, informais e individuais. Nestes editais, os critérios de priorização mencionados foram àqueles estabelecidos pela Resolução CD/FNDE ${ }^{\circ}$ 26/2013. Os grupos formais, inclusive um grupo de assentados da reforma agrária, formalizaram contrato com a prefeitura, mas nenhum deles eram fornecedores locais (Resolução CD/FNDE n 26, 2013). Os agricultores de âmbito local tiveram participação apenas nos editais de 2018 a 2020 (Tabela 2).

Destaca-se que nos editais de CP não foi explicitado o conceito de territórios rurais, o que pode gerar dificuldade na compreensão por parte dos agricultores familiares quanto ao grupo de projetos de vendas que fará parte no processo seletivo. Essa importante lacuna nas CP pode ser decorrente do fato de que a Lei $\mathrm{n}^{\circ}$ 11.947/2009 e as resoluções subsequentes que o operacionalizam também não contém a definição de territórios rurais, impossibilitando que os setores responsáveis pela seleção dos projetos de venda elejam de forma precisa os produtores situados nesses territórios.

Nos editais publicados entre 2010 e 2015, foram utilizados como critério de seleção o menor preço dos produtos ofertados pelos agricultores, ou seja, o primeiro classificado seria aquele que apresentasse o menor valor. Isso evidencia o descumprimento da legislação pertinente.

A Lei $n^{\circ} 11.947 / 2009$, ao instituir que a aquisição de alimentos da AF ocorra dispensando-se o processo licitatório regular, propõe incentivar e desenvolver o meio rural garantindo a participação dos pequenos agricultores num mercado institucional, que, caso essa dispensa não fosse instituída, os pequenos produtores não teriam condições de acessar o programa, devido à concorrência com grandes proprietários (Lei nº 11.947, 2009; Messias, 2018).

Para tanto, a legislação também estabelece a definição e acato a proposição de preço justo que, no caso dos agricultores familiares, devem ser definidos observando-se os custos de produção, transporte e distribuição, tomando como referência os preços praticados nas feiras de agricultores familiares locais, conforme estipulado no artigo 23 da Resolução CD/FNDE nº 38/2009 e artigo 29 da Resolução CD/FNDE n 26/2013 (Resolução CD/FNDE n 38, 2009; Resolução CD/FNDE nº 26, 2013), determinação acatada pelo município a partir de 2016. 
Entre 2016 e 2019, todos os editais utilizaram como critérios de seleção àqueles estabelecidos pela Resolução CD/FNDE $n^{\circ} 4 / 2015$, porém, em nenhum deles estava explícita esta resolução como referência, conforme descrito anteriormente. Somente a Resolução CD/FNDE n 26/2013 foi citada nestes editais, no entanto, a organização de critérios de seleção descrita contrariava esta normativa, pois foi instituída pela Resolução CD/FNDE n ${ }^{\circ}$ 4/2015. A descrição correta dos critérios de seleção e priorização, com base na legislação vigente, ocorreu apenas no edital do ano 2020. Alguns estudos apontam que esse fato pode ser decorrente da falta de capacitação e a dificuldade dos atores envolvidos em lidar com os processos de CP (Castellani et al., 2017; Rockett et al., 2019).

Quanto ao critério de vulnerabilidade social, percebe-se que ainda é restrito o envolvimento desses grupos na execução do programa. Observou-se que a participação de assentados da reforma agrária no fornecimento dos gêneros alimentícios ao PNAE, iniciou somente no ano 2015, por meio de uma cooperativa não localizada no município estudado (Tabela 2). No entanto, desde o ano 2000, há assentamentos cadastrados no município e poderiam estar inseridos nos processos de CP (Instituto Nacional de Colonização e Reforma Agrária [INCRA], 2017).

Tabela 2. Características dos fornecedores contratados, São Cristóvão/SE, 2010-2020.

\begin{tabular}{|c|c|c|c|c|c|c|c|c|c|c|c|c|c|c|}
\hline \multirow[b]{2}{*}{ Ano } & \multirow[b]{2}{*}{$\begin{array}{c}\mathrm{N}^{\mathrm{o}} \\
\text { contratos }\end{array}$} & \multicolumn{4}{|c|}{$\begin{array}{c}\text { Procedência dos } \\
\text { fornecedores }\end{array}$} & \multicolumn{3}{|c|}{$\begin{array}{c}\text { Comunidades } \\
\text { vulneráveis }\end{array}$} & \multicolumn{3}{|c|}{$\begin{array}{l}\text { Modos de } \\
\text { produção }\end{array}$} & \multicolumn{3}{|c|}{$\begin{array}{c}\text { Modalidade de } \\
\text { participação }\end{array}$} \\
\hline & & Local & $\mathrm{TR}$ & Estado & País & ARA & Quil. & Ind. & Org. & Agro. & Conv. & Formal & Informal & Individual \\
\hline 2010 & 0 & - & - & - & - & - & - & - & - & - & - & - & - & - \\
\hline 2011 & 0 & - & - & - & - & - & - & - & - & - & - & - & - & - \\
\hline 2012 & 3 & - & - & 3 & - & - & - & - & - & - & - & 3 & - & - \\
\hline 2013 & 3 & - & - & 3 & - & - & - & - & - & - & - & 3 & - & - \\
\hline 2014 & 4 & - & - & 4 & - & & - & - & - & - & - & 4 & - & - \\
\hline 2015 & 1 & - & - & 1 & - & 1 & - & - & - & - & - & 1 & - & - \\
\hline 2016 & 5 & - & - & 5 & - & 1 & - & - & - & - & - & 5 & - & - \\
\hline 2017 & 8 & - & - & 8 & - & 2 & - & - & - & - & - & 8 & - & - \\
\hline 2018 & 10 & 4 & - & 6 & - & 5 & - & - & - & - & - & 6 & - & 4 \\
\hline 2019 & 9 & 2 & - & 7 & - & 5 & - & - & - & - & - & 7 & - & 2 \\
\hline 2020 & 10 & 2 & - & 8 & - & 5 & - & - & - & - & - & 9 & - & 1 \\
\hline $\begin{array}{l}\text { Notas: } \\
\text { O traç } \\
\text { ARA - } \\
\text { Agro- } \\
\text { Conv. } \\
\text { Ind. - } \\
\text { Org. - } \\
\text { Quil. - } \\
\text { TR- Te } \\
\text { Fonte: }\end{array}$ & $\begin{array}{l}\text { o (-) signific } \\
\text { - Assentados } \\
\text { - Agroecoló } \\
\text { - Convencio } \\
\text { Indígenas } \\
\text { Orgânicos } \\
\text { Quilombola } \\
\text { erritórios Ru } \\
\text { Autoras. }\end{array}$ & $\begin{array}{l}\text { ca que a } \\
\text { s da Ref } \\
\text { gicos } \\
\text { onal } \\
\\
\text { las } \\
\text { urais }\end{array}$ & $\begin{array}{l}\text { inform } \\
\text { orma }\end{array}$ & $\begin{array}{l}\text { lação não } \\
\text { Agrária }\end{array}$ & foi en & ontrada & nos con & tratos. & & & & & & \\
\hline
\end{tabular}

Em um levantamento realizado pelo Instituto Nacional de Colonização e Reforma Agrária (INRA) (2017), observouse que no município de São Cristóvão/SE há quatro assentamentos e 154 famílias assentadas que trabalham na AF com plantação de hortaliças, mandioca, inhame, feijão, milho, dentre outras (INCRA, 2017). No entanto, a participação desses produtores assentados do município, ocorreu discretamente a partir de 2018, de forma individual (Tabela 2). Em anos subsequentes, os agricultores assentados organizados em grupos formais e residentes em outros municípios do estado também forneceram seus produtos ao programa.

O estado de Sergipe possui 243 assentamentos e 10.972 famílias assentadas, conforme dados do INCRA relativos ao 
ano de 2017. Este estado também possui 37 comunidades quilombolas certificadas pela Fundação Palmares, 18 destas tituladas pelo INCRA (INCRA, 2017). Em São Cristóvão não há cadastro de comunidades quilombolas e indígenas.

Esses resultados corroboram com o estudo realizado por Rodrigues et al. (2017), que caracterizou a inserção da AF na alimentação escolar no município de Alegre-ES no período de 2013 a 2015. Eles observaram que em 2015, das nove associações rurais (grupos formais) do município que participaram da $\mathrm{CP}$, apenas uma era de agricultores familiares assentados, demonstrando a baixa inserção deste no mercado institucional.

A inserção das comunidades vulneráveis no PNAE é de grande relevância para a geração de trabalho e renda, a redução do êxodo rural e o desenvolvimento dessas comunidades, uma vez que essas minorias sociais comumente vivem em condições crônicas de vulnerabilidade e precariedade socioeconômica (Leal, 2013; Ministério do Desenvolvimento Agrário [MDA], 2018).

Em relação ao critério modo de produção sustentável, não foi observada a descrição da aquisição desses gêneros pela prefeitura em todo o período analisado (Tabela 2). Na expectativa de encontrar algum gênero produzido certificado como agroecológico ou orgânico, analisou-se a especificação dos produtos nos contratos de venda, no entanto, na relação dos mesmos não constava esta informação.

Um estudo realizado por Santos e Souza (2014), em 2011, identificou que na região Leste de Sergipe (onde está situado o município de São Cristóvão), haviam 16 agricultores familiares com produção de base orgânica. Estes agricultores, por ser um grupo prioritário no processo de $\mathrm{CP}$, embora não residissem no município, poderiam estar inseridos no programa, na condição de grupo de projetos de abrangência estadual (Resolução CD/FNDE nº 4, 2015).

Destaca-se a dificuldade de obtenção de dados relativos ao quantitativo de agricultores familiares de base orgânica e agroecológica do município de São Cristóvão/SE. Foram realizadas inúmeras buscas no site da prefeitura do município, no site do IGBE, Ministério da Agricultura, Pecuária e Abastecimento (MAPA), MDA e Empresa de Desenvolvimento Agropecuário de Sergipe (EMDAGRO), entretanto, não foram encontrados registros sobre o quantitativo.

A falta de fornecedor orgânico e agroecológico para a alimentação escolar, embora haja produção no município, foi o principal motivo relatado pela RT para a não aquisição desses produtos nas CP. Uma das principais causas relatadas está relacionada a dificuldade de certificação dos gêneros e a falta de assistência técnica. Essas e outras causas, como a relação custo-benefício, a falta de articulação entre os agricultores e os responsáveis pelo processo de $\mathrm{CP}$, a logística e atrasos nos pagamentos por parte das EEx, tem sido relatadas em inúmeras evidências (Castellani et al., 2017; Gregolin et al., 2017; Rockett et al., 2019; Rodrigues et al., 2017; Santos et al., 2014; Silva \& Souza, 2013; Souza-Esquerdo \& Bergamasco, 2014; Triches et al., 2019; Triches \& Schneider, 2010).

Salienta-se que mesmo havendo dificuldades na comercialização desses gêneros, é importante fortalecer a prática de sua aquisição no âmbito do PNAE, utilizando-se das prioridades previstas na legislação (Resolução CD/FNDE no 4, 2015). A não priorização de grupos fornecedores que possuem produção de base orgânica e agroecológica, descumpre a recomendação legal do PNAE, contrasta com o pilar ambiental da sustentabilidade (Santos \& Souza, 2014) e impacta de forma negativa na dimensão qualitativa do DHAA.

No que se refere ao critério modalidade de participação dos agricultores familiares, em conformidade com a legislação, nas CP realizadas entre 2012 e 2017 do município estudado, houve somente a contratação de grupos formais, localizados em outros municípios do estado, aumentando gradativamente ao longo dos anos (Tabela 2). Em 2020, uma associação do município estudado foi contratada. Esses achados corroboram com o estudo realizado por Rodrigues et al. (2017) no município de Alegre-ES, que demonstraram a participação desses grupos durante todo o período analisado.

As cooperativas e associações, devido as suas condições de organização e posse de maior capital de giro, possibilitam uma estrutura de armazenamento e transporte mais adequada, possuem menor dificuldade em atender aos aspectos 
burocráticos, favorecendo a capacidade de oferta de forma regular ao programa (Cunha, 2015). Ao priorizar os grupos formais de agricultores, o PNAE contribui no fortalecimento de organizações dos pequenos produtores e favorece maior inserção destes no mercado institucional, contribuindo para a garantia da SAN dessas famílias (Assis et al., 2019) e fomento da economia local.

Baccarin et al. (2017) ressaltaram a importância das cooperativas na intermediação da participação dos agricultores no PNAE, mediante cooperação em diversos aspectos para o acesso à política. Por outro lado, Marques et al. (2014) inferiram que fazer parte de um grupo formal exige a necessidade de compreensão quanto aos interesses comuns, a coletividade, confiança, diálogo e companheirismo, o que leva alguns agricultores familiares a preferirem o fornecimento dos produtos de forma individualizada.

Destaca-se que o processo de CP realizadas no ano de 2020, ocorreu de forma atípica, pois, devido à pandemia do Covid-19, todas as redes de ensino suspenderam as aulas e as atividades presenciais nas escolas de todo o Brasil. Assim, os gestores adotaram diferentes medidas para não interromper a aquisição dos gêneros da $\mathrm{AF}$ e o fornecimento destes aos escolares (Amorim, Ribeiro-Junior \& Bandoni, 2020).

Com a publicação da Lei $\mathrm{n}^{\circ}$ 13.987/2020, ficou autorizado que em caráter excepcional, durante o período de suspensão das aulas, deveria ocorrer a distribuição de gêneros alimentícios adquiridos com recursos do PNAE aos pais ou responsáveis dos estudantes das escolas públicas da educação básica (Lei n ${ }^{\circ}$ 13.987, 2020). Logo após, o FNDE publicou a Resolução ${ }^{\circ}$ 2, que dispõe sobre a execução do PNAE durante a calamidade pública, reforçando que, no processo de aquisição dos produtos da AF priorizasse, sempre que possível, a compra local. Não houve alteração nos critérios de seleção nem de priorização dos grupos de projetos de venda (Resolução ${ }^{\circ}$ 02, 2020).

Por se tratar de um estudo de caso, e considerando as especificidades socioeconômicas, políticas, culturais e ambientais do lócus de pesquisa, reconhece-se a impossibilidade de inferências generalizadas, que ultrapassem os limites da interpretação dos resultados condicionados as peculiaridades do universo de pesquisa.

\section{Conclusão}

Os resultados do presente estudo confirmam a hipótese elaborada. Os agricultores de abrangência local tiveram menor participação que os demais grupos, segundo procedência, no processo de seleção dos projetos de vendas. Essa situação compromete a possibilidade de desenvolvimento local, a garantia de trabalho e renda, e a promoção da agricultura local através do PNAE.

A análise das CP permitiu identificar que os atores envolvidos na elaboração destas apresentam dificuldades em compreender e seguir a legislação pertinente, assim como, entender a relevância dela para a execução das compras do PNAE. Sugere-se que sejam realizadas ações junto à universidade, com articulações intersetoriais e com os diversos atores envolvidos na aquisição dos produtos da $\mathrm{AF}$, inclusive os produtores, visando capacita-los e habilitá-los para o processo de $\mathrm{CP}$.

Ademais, enfatiza-se a necessidade da assistência técnica no município para que os entraves referentes aos aspectos burocráticos e de produção dos gêneros alimentícios supracitados possam ser minimizados.

Devido à falta de certificação ser um problema enfrentado pelos agricultores familiares de base orgânica e agroecológica em ofertar seus produtos, é preciso criar mecanismos que facilitem o acesso dos produtores ao processo de certificação para facilitar a participação destes no programa.

Sugere-se que novos estudos sejam realizados visando o mapeamento da produção orgânica e agroecológica no município, para identificar a localização, o quantitativo de agricultores familiares e os fatores que impedem a participação destes nas $\mathrm{CP}$. 


\section{Referências}

Aguiar, J. A., \& Calil, R. M. (2018). Análise da adequação normativa de chamadas Públicas para compra de alimentos dos agricultores familiares destinados ao Programa Nacional de Alimentação escolar (PNAE). Hig Aliment, 32, (278/279), 22-29.

Alexandre, V. P., Gomes, L. O. F., Silva, S. U, Almeida, G. M., Martins, K. A., Monego, E. T., Sousa, L. M., \& Campos, M. R. H. (2016). Do campo à escola: compra de alimentos da agricultura familiar pelo Programa Nacional de Alimentação Escolar em Territórios da Cidadania de Goiás. Segur Alim Nutric, 23, 1049-1064.

Amorin, A. L. B., Ribeiro-Junior, J. R. S., \& Bandoni, D. R. (2020). Programa Nacional de Alimentação Escolar: estratégias para enfrentar a insegurança alimentar durante e após a COVID-19. Revista De Administração Pública, 54(4):1134-1145.

Andrade, J. C., Araujo, M. A., \& Santos, P. V. S. (2019). Estudo sobre aquisição de alimentos da agricultura familiar para o programa nacional de alimentação escolar. Gestão e Socied., 13(36), 3062-3089.

Assis, T. R. P., França, A. G. M., Coelho, A. M. (2019). Agricultura familiar e alimentação escolar: desafios para o acesso aos mercados institucionais em três municípios mineiros. Rev de Econ Sociol Rural, 57(4), 577-593.

Baccarin, J. P., Triches, R. M., Teo, R. P. A., \& Silva, D. B. P. (2017). Indicadores de Avaliação das Compras da Agricultura Familiar para Alimentação Escolar no Paraná, Santa Catarina e São Paulo. Rev Econ Sociol Rural, 55(1), 103-122.

Brito, T. P., Pereira, V. G., \& Santos, A. P. (2018). Os limites, desafios e Potencialidades da agricultura Familiar no PNAE em municípios Atingidos pela mineração. Retratos de Assentados, 21(2), 192-224.

Castellani, A. L. A., Trentini, T., Nishida, W., Rossi, C. E., Costa, L. C. F., \& Vasconcelos, F. A. G. (2017). Purchase of family farm and organic foods by the Brazilian School Food Program in Santa Catarina state, Brazil. Rev Nutri, 30(5), 651-662.

Costa, S. L. (2016). Áreas protegidas e assentamentos rurais em sergipe: sob o enfoque do princípio constitucional do desenvolvimento sustentável (Tese de Doutorado). Universidade Federal de Sergipe, São Cristóvão, Brasil.

Cunha, W. A. (2015). Efeitos dos programas governamentais de aquisição de alimentos para a agricultura familiar no contexto local. (Dissertação de Mestrado). Universidade Federal de Viçosa (UFV). Viçosa (MG), Brasil.

Cunha, W. A., Freitas, A. F., \& Salgado, R. J. S. F. (2017). Efeitos dos Programas Governamentais de Aquisição de Alimentos para a Agricultura Familiar em Espera Feliz, MG. Rev Econ Soc Rural, 55(3), 427-444.

Dorneles, F. M., Mainardi, C. F., Bettencourt, A. F., Pires, R. G., Simões, F. S. B., \& Nascimento, S. G. S. N. (2018). Panorama da aquisição de alimentos da Agricultura Familiar para o Programa Nacional de Alimentação Escolar em um município do Rio Grande do Sul. ECoDAF, 4(1), 86-101.

Flick, U. (2009). Introdução à pesquisa qualitativa. Artmed, 3, 402.

Gonçalves, H. V. B., Cunha, D. T., Stedefeldt, E., \& Rosso, V. V. (2015). Family farming products on menus in school feeding: a partnership for promoting healthy eating. Ciência Rural, 45(12), 2267-2273.

Gregolin, M. R. P, Santos, C. S., Felippini, M. L., Mateus, M. A. F., \& Christoffo, P. I. (2017). Potencialidades e Fragilidades do Programa Nacional de Alimentação Escolar - PNAE no Território Cantuquiriguaçu (PR). Conexao UEPG, 13(3), 548-567.

Instituto Brasileiro de Geografia e Estatística. (2018). Trabalho e rendimento Autor.

Instituto Brasileiro de Geografia e Estatística. (2003). Mapa de pobreza e desigualdade. Autor.

Instituto Brasileiro de Geografia e Estatística. (2010). Censo demográfico. Autor.

Instituto Brasileiro de Geografia e Estatística. (2010). Índice de Desenvolvimento Humano Municipal (IDHM). Autor.

Instituto Brasileiro de Geografia e Estatística. (2017). Censo Agropecuário de 2017. Autor.

Instituto Brasileiro de Geografia e Estatística. (2018). Produto Interno Bruto (PIB). Autor.

Instituto Brasileiro de Geografia e Estatística. (2019). Produção Agrícola Municipal. Autor.

Instituto Brasileiro de Geografia e Estatística. (2020). Estimativas da população residente com data de referência de julho de 2017. Autor.

Instituto Nacional de Colonização e Reforma Agrária. (2017). Incra nos Estados: Informações gerais sobre os assentamentos da Reforma Agrária. Autor.

Instituto Nacional De Colonização E Reforma Agrária. (2017). Relação de Beneficiários do Programa Nacional de Reforma Agrária (PNRA). Autor.

Leal, S. C. T. O. (2013). Programa de Aquisição de Alimentos (PAA) e a Disputa territorial em torno da luta pela/na terra no pontal do Paranapanema - SP (Monografia). Faculdade De Ciências E Tecnologia, Presidente Prudente, Brasil.

Lei $\mathrm{n}^{\circ} 11.947$, de 16 de junho de 2009. (2009). Dispõe sobre o atendimento da alimentação escolar e do Programa Dinheiro Direto na Escola aos alunos da educação básica. Brasília, DF. http://www.planalto.gov.br/ccivil_03/_Ato2007-2010/2009/Lei/L11947.htm

Lei $\mathrm{n}^{\circ}$ 12.527, De 18 De Novembro De 2011. (2011). Regula o acesso a informações previsto no inciso XXXIII do art. $5^{\circ}$, no inciso II do $\S 3^{\circ}$ do art. 37 e no $\S$ $2^{\circ}$ do art. 216 da Constituição Federal; altera a Lei ${ }^{\circ}$ 8.112, de 11 de dezembro de 1990; revoga a Lei $\mathrm{n}^{\circ} 11.111$, de 5 de maio de 2005 , e dispositivos da Lei ${ }^{\circ}$ 8.159, de 8 de janeiro de 1991; e dá outras providências. Brasília, DF. http://www.planalto.gov.br/ccivil_03/_ato2011- 
2014/2011/lei/112527.htm\#: :text=Regula\%20o\%20acesso\%20a\%20informa\%C3\%A7\%C3\%B5es\%20previsto\%20no\%20inciso\%20XXXIII\%20do\%20art.\& text $=216 \% 20 \mathrm{da} \% 20 \mathrm{Constitui} \% \mathrm{C} 3 \% \mathrm{~A} 7 \% \mathrm{C} 3 \% \mathrm{~A} 3 \mathrm{o} \% 20 \mathrm{Federal} \% 3 \mathrm{~B} \% 20$ altera, $1991 \% 3 \mathrm{~B} \% 20 \mathrm{e} \% 20 \mathrm{~d} \% \mathrm{C} 3 \% \mathrm{~A} 1 \% 20 \mathrm{outras} \% 20 \mathrm{provid} \% \mathrm{C} 3 \% \mathrm{AAncias}$

Lei $\mathrm{n}^{\circ}$ 13.987, de 7 de abril de 2020. (2020) Altera a Lei $\mathrm{n}^{\circ}$ 11.947, de 16 de junho de 2009, para autorizar, em caráter excepcional, durante o período de suspensão das aulas em razão de situação de emergência ou calamidade pública, a distribuição de gêneros alimentícios adquiridos com recursos do Programa Nacional de Alimentação Escolar (Pnae) aos pais ou responsáveis dos estudantes das escolas públicas de educação básica. Brasília, DF. https://www.in.gov.br/en/web/dou/-/lei-n-13.987-de-7-de-abril-de-2020-251562793?inheritRedirect=true\&redir ect=\%2Fweb\%2F guest\%2Fsearch \%3FqSea rch\%3D13.987\%252C\%2520de\%25207\%2520de\%2520abril\%2520de\%25202020.

Lei $\mathrm{n}^{\circ}$ 8.666, de 21 de junho de 1993. (1993). Regulamenta o art. 37, inciso XXI, da Constituição Federal, institui normas para licitações e contratos da Administração Pública e dá outras providências. Brasília, DF. http://www.planalto.gov.br/ccivil_03/leis/18666cons.htm.

Marques, A. A., Fernandes, M. G. M., Leite, I. N., Viana, R. T., Gonçalves, M. C., \& Carvalho, A. T. (2014). Reflexões de agricultores familiares sobre a dinâmica de fornecimento de seus produtos para a alimentação escolar: o caso de Araripe, Ceará. Saude soc. [online], 23 (4), 1329-1341.

Messias, S. L. (2018). A chamada pública como alternativa à licitação: seu uso na quisição de produtos da agricultura familiar para a alimentação escolar (Dissertação de Mestrado). Faculdade de Ciências Humanas e Sociais, Franca, Brasil.

Ministério do Desenvolvimento Agrário. (2018). Agricultura familiar do Brasil é $8^{\mathrm{a}}$ maior produtora de alimentos do mundo. Brasil: Autor.: http://www.mda.gov.br/sitemda/noticias/agricultura-familiar-do-brasil-\%C3\%A9-8\%C2\%AA-maior-produtora-de-alimentos-do-mundo.

Mossmann, M. P., Teo, C. R. P. A., Busato, M. A., \& Triches, R. M. (2017). Interface between family farming and school feeding: barriers and coping mechanisms from the perspective of different social actors in Southern Brazil. Rev Econ Soc Rural, 55(2), 325-342.

Noronha, A. P. (2013). O acesso dos agricultores familiares prioritários ao Programa Nacional de Alimentação Escolar (PNAE): o caso do município de Capão do Leão, RS (Dissertação de Mestrado). Universidade Federal de Pelotas, Pelotas, Brasil.

Pereira, A. S., Shitsuka, D. M., Parreira, F. J. \& Shitsuka, R. (2018). Metodologia da pesquisa científica. Universidade Federal de Santa Maria. https://repositorio.ufsm.br/bitstream/handle/1/15824/Lic_Computacao_Metodologia-Pesquisa-Cientifica.pdf?sequence=1.

Prodanov, C. C., \& Freitas, E. C. (2013). Metodologia do Trabalho científico (recurso eletrônico): métodos e técnicas da pesquisa e do trabalho acadêmico. Novo Hamburgo, Feevale.

Resolução CD/FNDE/MEC no 2, de 9 de abril de 2020. (2020). Dispõe sobre a execução do Programa Nacional de Alimentação Escolar (PNAE) durante o período de estado de calamidade pública, reconhecido pelo Decreto Legislativo $\mathrm{n}^{\circ} 6$, de 20 de março de 2020, e da emergência de saúde pública de importância internacional decorrente do novo coronavírus (Covid-19). Brasília, DF.

Resolução do Conselho Deliberativo do Fundo Nacional de Desenvolvimento da Educação n. ${ }^{\circ}$ 4, de 3 de abril de 2015. (2015). Altera a redação dos artigos 25 a 32 da Resolução/CD/FNDE n. ${ }^{\circ}$ 26, de 17 de junho de 2013, no âmbito do Programa Nacional de Alimentação Escolar (PNAE). Brasília, DF. http://www.gestaoescolar.diaadia.pr.gov.br/arquivos/File/alimenatacao_escolar/resolucao042015_ceae.pdf.

Resolução $\mathrm{n}^{\circ}$ 10, de 17 de outubro de 2018. Dispõe sobre soluções garantidoras de direitos humanos e medidas preventivas em situações de conflitos fundiários coletivos rurais e urbanos. https://www.in.gov.br/web/dou/-/resolucao-n-10-de-17-de-outubro-de-2018-46888055

Resolução ${ }^{\circ}$ 6, de 8 de maio de 2020. (2020). Dispõe sobre o atendimento da alimentação escolar aos alunos da educação básica no âmbito do Programa Nacional de Alimentação Escolar - PNAE. Brasília, DF. https://www.in.gov.br/en/web/dou/-/resolucao-n-6-de-8-de-maio-de-2020-256309972.

Resolução/CD/FNDE n 26, de 17 de junho de 2013. (2013). Dispõe sobre o atendimento da alimentação escolar aos alunos da educação básica no âmbito do Programa Nacional de Alimentação Escolar - PNAE. Brasília, DF. https://www.fnde.gov.br/index.php/acesso-ainformacao/institucional/legislacao/item/4620-resolu\%C3\%A7\%C3\%A3o-cd-fnde-n\%C2\%BA-26,-de-17-de-junho-de-2013

Resolução/CD/FNDE nº 38, de 16 de julho de 2009. (2009). Dispõe sobre o atendimento da alimentação escolar aos alunos da educação básica no Programa Nacional de Alimentação Escolar (PNAE). Diário Oficial da União, 2009. https://www.fnde.gov.br/index.php/acesso-ainformacao/institucional/legislacao/item/3341-resolu\%C3\%A7\%C3\%A3o-cd-fnde-n\%C2\%BA-38-de-16-de-julho-de-2009

Rockett, F. C., Corrêa, R. S., Pires, G. C., Machado, L. S., Hoerlle, F. S., Souza, C. O. M., \& Oliveira, A. B. A. (2019). Family farming and school meals in Rio Grande do Sul, Brazil. Ciência Rural, 49(2), 1-12.

Rodrigues, R., Siqueira, H. M., Biancardi, C. C. S., Andrade, M. A. N., Valente, M. V., \& Paula, L. B. (2017). A aquisição de alimentos da agricultura familiar pelo PNAE no município de Alegre-ES. Demetra, 12(1), 91-112.

Santos, A. C. (2018). Diagnóstico situacional e proposta para a implementação do sistema nacional de segurança alimentar e nutricional em São Cristóvão, Sergipe. (Dissertação de Mestrado). Universidade Federal de Sergipe, São Cristóvão, Brasil.

Santos, C. O., \& Souza, R. M. (2014). Territórios produtivos da agricultura orgânica em Sergipe. Revista de Geografia (UFPE). 31 (1), $58-72$.

Santos, F., Fernandes, P. F., Rockett, F. C., \& Oliveira, A. B. A. (2014). Avaliação da inserção de alimentos orgânicos provenientes da agricultura familiar na alimentação escolar, em municípios dos territórios rurais do Rio Grande do Sul, Brasil. Ciênc. saúde coletiva 2014, 19(5), 1429-1436.

Saraiva, E. B., Silva A. P. F., Sousa, A. A., Cerqueira, G. F., Chagas, C. M. S., \& Toral, N. (2013). Panorama da compra de alimentos da agricultura familiar para o Programa Nacional de Alimentação Escolar. Ciênc Saúde Colet, 18(4), 927-935.

Silva, A. P. F., \& Souza, A. A. (2013). Alimentos orgânicos da agricultura familiar no Programa Nacional de alimentação Escolar do Estado de Santa Catarina, Brasil. Rev Nutr, 26(6), 701-714.

Silva, L. R. C., Damaceno, A. D., Martins, M. C. R., Sobral, K. M., \& Farias, I. M. S. (2009, outubro). Pesquisa Documental: Alternativa Investigativa na Formação Docente. Trabalho apresentado no IX Congresso Nacional de educação, Paraná. 
Research, Society and Development, v. 10, n. 4, e26610414028, 2021

(CC BY 4.0) | ISSN 2525-3409 | DOI: http://dx.doi.org/10.33448/rsd-v10i4.14028

Silva, S. S., Alves J., A., Freitas, A. F., \& Freitas, A. F. (2017). A interface entre alimentação escolar e agricultura familiar em Jampruca e Mathias Lobato, Minas Gerais (Brasil). Revista Espacios, 38 (46), 1-14.

Sodré, M. T., \& Salamoni, G. (2016). A coexistência do pensamento cartesiano e sistêmico: As limitações territoriais enfrentadas pelo PAA e PNAE em Pelotas/RS. Socied Nat., 28 (3), 457-471.

Souza, L. B. B. (2012). Organizações da agricultura familiar no Estado de São Paulo e sua experiência de fornecimento para o PNAE. In: Corá MAJ, Belik W, (org). Projeto Nutre SP - análise da inclusão da agricultura familiar na alimentação escolar no estado de São Paulo (p.29-45). Instituto Via Pública.

Souza-Esquerdo, V. F., \& Bergamasco, S. M. P. P. (2014). Análise sobre o acesso aos programas de políticas públicas da agricultura familiar nos municípios do Circuito das Frutas (SP). Rev Econ Soc Rural, 52(1), 205-222.

Triches, R. M., Barbosa, L. P., \& Silvestri, F. (2016). Agricultura Familiar e Alimentação Escolar no Estado do Paraná: uma análise das chamadas públicas. Rev paranaense de desenvolvimento, 37(130), 29-43.

Triches, R. M., \& Schneider S. (2010). Alimentação escolar e agricultura familiar: reconectando o consumo à produção. Saúde Soc., 19 (4), $933-45$.

Triches, R. M., Simonetti, M. G., Cessarino, J. P., Baccarin, J. G., \& Teo, C. R. P. A. (2019). Condicionantes e limitantes na aquisição de produtos da agricultura familiar pelo Programa de Alimentação Escolar no estado do Paraná. Redes, 24(1), 118-137.

Ventura, M. M. (2007). O Estudo de Caso como Modalidade de Pesquisa. Rev SOCERJ, 20(5), 383-386.

Vilela, K. F., Freitas, A. F., Barbosa, R. A., \& Salgado, R. J. S. F. (2019). Implementation of the national school nourishment program in the Brazilian Federal institution of Education of Bahia State. Cienc. Rural [online], 49 (9), 1-11. 